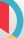

\title{
Generalized Anxiety Disorder 7-Item Scale and Templer's Death Anxiety Scale in Iranian Inpatients with COVID-19: Evaluation of Psychometric Properties and Diagnostic Accuracy
}

\author{
Abolfazl Fattah (iD ${ }^{1}$, Abbas Ebadi (iD) ${ }^{2}$, Narmin Borumand ${ }^{3}$, Abdolhadi Saeedi ${ }^{4}$, Mahdieh Darbani ${ }^{1}$, \\ Sona Setayesh ${ }^{1}$, Gholamreza Mohammadi ${ }^{1}$ and Masoudeh Babakhanian (iD) ${ }^{5, *}$ \\ ${ }^{1}$ Velayat Hospital, Semnan University of Medical Sciences, Semnan, Iran \\ ${ }^{2}$ Behavioral Sciences Research Center, Life Style Institute, Nursing Faculty, Baqiyatallah University of Medical Sciences, Tehran, Iran \\ ${ }^{3}$ Shiraz branch, Islamic Azad University, Shiraz, Iran \\ ${ }^{4}$ Urmia university of Medical sciences, Mahabad, Urmia, Iran \\ ${ }^{5}$ Student Research Committee, Psychiatry and Behavioral Research Centre, Addiction Institute, Mazandaran University of Medical Sciences, Sari, Iran \\ "Corresponding author: Student Research Committee, Psychiatry and Behavioral Research Centre, Addiction Institute, Mazandaran University of Medical Sciences, Sari, Iran. \\ Email: babakhanian.m@gmail.com
}

Received 2020 November 18; Revised 2021 July 27; Accepted 2021 July 28.

\begin{abstract}
Background: During the outbreak of the coronavirus disease 2019 (COVID-19) pandemic, anxiety has always been with patients. Objectives: It is necessary to obtain a valid instrument for the evaluation and screening of patients with anxiety. The current study attempted to evaluate the sensitivity and specificity and provide the clinical cut-off scores for the Death Anxiety Scale (DAS) and Generalized Anxiety Disorder 7-Item (GAD-7) Scale in hospitalized patients diagnosed with COVID-19 in Iran.

Methods: This diagnostic accuracy study was carried out for 4 months on 150 hospitalized patients diagnosed with COVID-19 to evaluate generalized anxiety disorder and death anxiety. Cronbach's alpha was used to evaluate reliability. For the determination of the presence of anxiety disorders, the Structured Clinical Interview with hospitalized subjects was conducted by a psychiatrist based on the Diagnostic and Statistical Manual of Mental Disorders Fourth Edition diagnostic criteria.

Results: The Cronbach's alpha coefficients for the GAD-7 Scale and DAS questionnaires were observed to be 0.88 and 0.74 , respectively, confirming their reliability. Based on cut-off scores with the best balance, the sensitivity and specificity of the GAD-7 Scale questionnaire were $61.9 \%$ and $86.9 \%$, respectively. Moreover, the sensitivity and specificity values related to the DAS questionnaire were $47.8 \%$ and $73.8 \%$, respectively. The values for the area under the curve were 0.75 and 0.63 for the GAD-7 Scale and DAS, respectively. With this scoring method, those who scored higher than 8 and 7 in the GAD-7 Scale and DAS questionnaires were considered patients, respectively. Moreover, in these two tests, higher levels of generalized anxiety and death anxiety were reported in female patients.

Conclusions: The GAD-7 Scale and DAS both showed adequate psychometric properties and diagnostic accuracy; therefore, they are applicable for anxiety screening in patients with COVID-19. It will likely take a few years to bring the virus under control worldwide. Iran's Ministry of Health should implement exact psychological interventions during hospitalization and after discharge to prevent the adverse mental health consequences of COVID-19.
\end{abstract}

Keywords: Anxiety, Death, COVID-19, Area Under the Curve, Reliability, Iran

\section{Background}

Emerging infectious diseases are among the great challenges of the 21st century. Originating in Wuhan, China, the new coronavirus disease has reached the level of a pandemic, affecting countries all across the globe (1). According to the Centers for Disease Control and Prevention, the incubation period for this unknown virus extends to 14 days. Since coronavirus is mainly transmitted via tiny respiratory droplets, it spread to almost every region of the globe in less than a month and caused great anxiety and panic in individuals (2). The lack of sufficient information or false information about the transmission of the new virus, its incubation period, the number of infected individuals, and its geographic reach gave rise to this panic and stress (2). Isolation affected many aspects of individuals' lives and triggered widespread mental health problems, such as panic, anxiety, and depression (3).

Social media and cyberspace can also be blamed for 
spreading exaggerating news about the risk and spread of the coronavirus, which reinforced this fear and panic (4). Coronavirus disease 2019 (COVID-19) instilled greater fear in individuals, compared to the seasonal flu, although the latter has killed a considerably larger number of individuals. According to a new Morning Consult survey (2020), $37 \%$ of Americans were worried about COVID-19; however, $27 \%$ were very concerned about the seasonal flu. In addition, most respondents (62\%) were more worried about severe acute respiratory syndrome coronavirus 2 rather than the seasonal flu (5).

Recently, fear of death has been shown to predict anxiety related to COVID-19. Fear of death has been observed to be predictive of COVID-19-related anxiety and contributive to different health conditions, including the suppression of anxiety-provoking thoughts and denial of one's vulnerability (6). The nature and impact of COVID-19 on mental health have not yet been fully understood. Therefore, further studies are needed to shed light on the relationship between generalized anxiety disorder and coronavirusrelated death anxiety. Coronavirus has occasioned expected expansions in death anxiety. Social separation, limited human relationships, and restricted contact with kins, guardians, and dear companions are among the primary causes of death anxiety (6).

Given all the above-mentioned issues, there are now two important questions, including whether health care systems worldwide are ready to deal with the patients' increasing anxiety and whether patients' concerns are assessed and controlled by mental health professionals to prevent psychosocial consequences. During previous epidemics, such as severe acute respiratory syndrome (SARS), the results of studies showed that mental health problems occurred. According to the evidence, the mental state of individuals can also be influenced by the COVID-19 pandemic $(7,8)$. Regardless of whether COVID-19 is a major epidemic (9), virologists predict that the next severe epidemic is inevitable and may occur in the coming years (10).

At the time of this study, there were no particular robust screening tools or diagnostic instruments to help promptly identify relevant symptoms and study the epidemiology of mental health problems (11) caused by COVID-19. Accordingly, it is necessary to re-standardize the instruments, such as the Generalized Anxiety Disorder 7Item (GAD-7) Scale and Death Anxiety Scale (DAS), in the target population. To date, in Iran, the Hospital Anxiety and Depression Scale about anxiety have been standardized (12). Moreover, the GAD-7 Scale and DAS questionnaires have been administered to different populations of hospitalized patients, such as cancer patients and those with epilepsy. However, for the first time in the world, a clinical incision point has been designed for COVID-19 inpatients.
This study may help to develop guidelines for the alleviation of individuals' anxiety during the COVID-19 pandemic in Iran.

\section{Objectives}

Previous studies suggest that a fear of death predicts anxiety about the coronavirus, which has been observed to cause deeper psychological distress in the patients (13). With this background and the above-mentioned objectives in mind, the present study aimed to evaluate the psychometric properties, sensitivity, and specificity and provide the clinical cut-off scores for the DAS and GAD-7 Scale in hospitalized patients diagnosed with COVID-19 in Iran.

\section{Methods}

This diagnostic accuracy study was carried out under the approval and financial support of the Student Research Committee of Mazandaran University of Medical Sciences, Sari, Mazandaran, Iran (IR.MAZUMS.REC.1399.131).

\subsection{Sample Population}

The current study was performed for 4 months on hospitalized patients with a definitive diagnosis of COVID-19. The statistical population included all COVID-19 patients throughout the country. Moreover, this study was exclusively conducted in the selected hospitals of West Azerbaijan and Semnan provinces (Velayat Hospital of Damghan, Iran, and Imam Khomeini hospitals of Mahabad, Iran). The inclusion criteria were diagnosis of COVID-19 as confirmed by the treating physician and based on lung computed tomography scan, familiarity with electronic equipment to be able to complete the questionnaires, and alertness or ability to participate in the study.

The exclusion criteria were incomplete questionnaires, a severe organic pulmonary disorder, and functional disability to take the test. According to similar studies, the prevalence of anxiety in patients with COVID19 admitted to hospitals is estimated to be $63 \%$ (14). The number of samples required for the minimum sample size for sensitivity and specificity test was calculated using PASS software (version 11) (15) as follows:

Prevalence of disease: 63\%; Ho = Null Hypothesis: 0.50; $\mathrm{H} 1$ = Hypothesis: 0.70; Minimum sample size $(\mathrm{n}=73)$; Maximum sample size $(n=122)$. Therefore, 150 samples were selected for this study. 


\subsection{Data Collection}

This psychometric and diagnostic accuracy study examined generalized anxiety disorder and death anxiety using the internet and social media. Anyone who used any kind of social network had access to the survey and was able to click on the link to complete the questionnaires, including a demographic information form, the general anxiety disorder questionnaire, and the death anxiety questionnaire developed by Templer.

The data were collected during May 2020 to August 2020. All the patients who had positive polymerase chain reaction results and were admitted to the hospital were identified every day by a psychologist. After obtaining informed consent from the patients, the examiner sent the download link of the questionnaire. A psychologist with a Master's degree in clinical psychology provided the hospitalized patients with the download link to the GAD-7 Scale and DAS questionnaires. The participants could withdraw from the study at any time without any justification. It is worth noting that the text of the questionnaire was provided on paper in case of non-access to the internet or social media. The psychiatrist interviewed each patient immediately after the completion of the questionnaire according to the Diagnostic and Statistical Manual of Mental Disorders Fourth Edition (DSM-IV) diagnostic criteria.

In this study, discriminant validity was assessed by calculating the operating characteristics. The internal consistency method (ie, Cronbach's alpha coefficient) was used to determine the reliability of the questionnaire. The obtained data were analyzed using SPSS software (version 24) and MedCalc software (20.0.13).

\subsection{Scales}

Researcher-Made Demographic Information Questionnaire: It sought the information related to the patients, including age, gender, educational level, underlying diseases, and history of mental illnesses.

GAD-7 Scale: It is a useful diagnostic tool developed to identify the probable cases of generalized anxiety disorder and measure the severity of clinical symptoms. This questionnaire has seven items. The responses include "not at all," "several days," "more than half the days", and "nearly every day," scored as $0,1,2$, and 3 , respectively. The total score of the GAD-7 Scale is within the range of $0-21$.

Spitzer et al. reported good internal consistency (0.92) and good test-retest reliability $(r=0.83)$ for the GAD-7 Scale. The sensitivity and specificity of the scale at the cut-off point of 10 or higher exceeds 0.80 (16). In a study conducted on 199 Iranian students to evaluate the psychometric properties of the GAD-7 Scale questionnaire, Cronbach's alpha coefficient of the test was observed to be 0.85 , and the test-retest reliability was calculated to be $r=0.48$ ( $P$ $=0.01$ ), showing the internal consistency and acceptable reliability of this scale in the Iranian population (17). The Persian version of this instrument was used in the current study.

Templer's DAS: This 15-item questionnaire, which was developed by Templer (1970) (18) nearly 50 years ago, has been the most commonly used scale for the measurement of death anxiety $(11,12)$. An extended version of this scale was published in 2006 with 51 items (13). In Iran, the internal consistency of the DAS was reported to be 0.75 in a study conducted by Tavakoli and Ahmadzadeh, who examined the validity and reliability of this 15 -item questionnaire administered to 345 students (14). The Persian version of this instrument was used in this study.

Structured Clinical Interview Based on DSM-IV Diagnostic Criteria: After completing the questionnaire, the Structured Clinical Interview for DSM-IV Axis I Disorders was used for diagnosis (19). During the past 6 months, the patients were assessed in terms of the presence of four anxiety disorders, namely general anxiety disorder, panic disorder, social phobia, and agoraphobia. Furthermore, the patients were asked whether they were currently under treatment for mental health problems or felt a need for treatment.

\subsection{Statistical Analysis}

\subsubsection{Reliability}

Cronbach's alpha was used to determine the internal consistency of the GAD-7 Scale and DAS questionnaires in SPSS software (version 24).

\subsubsection{Validity}

Two indicators were used to determine the validity of the GAD-7 Scale and DAS, namely, sensitivity (True Positive Rate) measuring the proportion of positives that are correctly identified and specificity (True Negative Rate) measuring the proportion of negatives that are correctly identified (20). For a test to be helpful, sensitivity plus specificity should be at least 1.5 (halfway between 1 , which is useless, and 2, which is perfect) (13).

Negative Predictive Value and Positive Predictive Value: These two terms are considered the proportion of positive and negative results in statistics and diagnostic tests that are true positive and true negative (21).

Positive Likelihood Ratio and Negative Likelihood Ratio: These two terms are used for assessing the value of performing a diagnostic test. They use the sensitivity and specificity of the test to determine whether a test result usefully changes the probability that a condition (eg, a disease state) exists (22). If the positive likelihood ratio (+LR) 
is higher than 10 or the negative likelihood ratio(-LR) is less than 0.1, it will help diagnose or rule out the disease (23).

Youden's Index: It is a single statistic that captures the performance of a dichotomous diagnostic test. Informedness refers to its generalization to the multi-class case and estimates the probability of an informed decision (24). To determine the best cut-off point between these two questionnaires, Youden's index was used with the following formula:

Youden's index defined as $\mathrm{J}=\operatorname{maxc}[\operatorname{Sensitivity}(\mathrm{c})+$ Specificity(c)-1] within the range of $0-1(25)$

\subsubsection{Diagnostic Odds Ratio}

The diagnostic odds ratio (DOR) measures the discriminative power of a diagnostic test or the ratio of the odds of a positive test result among the patients to the odds of a positive test result among the non-patients. This index significantly depends on the sensitivity and specificity of an experiment. The DOR calculation formula is as follows:

DOR = $\quad$ specificity/(1-sensitivity)/sensitivity/(1specificity) (26)

Furthermore, a receiver operating characteristic (ROC), curve was used to determine the optimal balance to determine the sensitivity and specificity and the areas under the ROC curve (AUC).

\section{Results}

\subsection{Demographic Information}

During the 4 months of the study, the participants included 150 patients with a definitive diagnosis of COVID-19 who had the willingness and ability to participate in the study. The mean age of the participants was $35.3 \pm 9.2$ years (range: 17 - 71). Furthermore, 60\% and 64\% of the female patients had generalized anxiety disorder and death anxiety, respectively. Moreover, $40 \%$ and $36 \%$ of the male patients had generalized anxiety disorder and death anxiety, respectively. Table 1 shows the demographic characteristics of the participants, and Table 2 shows the medical status history of the participants.

\subsection{Reliability}

According to the results, both the GAD-7 Scale and DAS have a high level of internal consistency since their alpha coefficients were higher than 0.7. In addition, the results of the analysis related to the convergent validity between the two tests pointed to a positive and acceptable correlation between the two instruments (Table 3).

\begin{tabular}{|c|c|}
\hline Characteristics & No. (\%) \\
\hline \multicolumn{2}{|l|}{ Gender } \\
\hline Male & $72(47)$ \\
\hline Female & $78(53)$ \\
\hline \multicolumn{2}{|l|}{ Educational level } \\
\hline Up to high school diploma & $103(68.6)$ \\
\hline Four-year university degree & $38(23.3)$ \\
\hline Postgraduate education & $9(6)$ \\
\hline \multicolumn{2}{|l|}{ Marital status } \\
\hline Married & $113(75.4)$ \\
\hline Single & $23(15.4)$ \\
\hline Divorced or widowed & $14(9.4)$ \\
\hline \multicolumn{2}{|l|}{ Occupation } \\
\hline Medical staff & $29(19.5)$ \\
\hline Nonmedical staff & $121(81.5)$ \\
\hline & No. (\%) \\
\hline \multicolumn{2}{|l|}{ Physical disorder } \\
\hline Asthma & $5(3.3)$ \\
\hline Heart disease & $1(0.6)$ \\
\hline Diabetes & $14(9.3)$ \\
\hline Blood pressure & $6(4)$ \\
\hline Cancer & $4(2.6)$ \\
\hline Kidney diseases & $1(0.6)$ \\
\hline \multicolumn{2}{|l|}{ History of psychological disorders } \\
\hline Anxiety disorders & $9(6)$ \\
\hline Mood disorders & $7(4.6)$ \\
\hline Drug and alcohol use disorders & $11(7.3)$ \\
\hline Sleep disorders & $7(4.6)$ \\
\hline
\end{tabular}

\subsection{Validity}

Discriminant Validity: As shown in Table 4, the values of the AUC were 75\% (indicating excellent discrimination) and $63 \%$ (showing acceptable discrimination) for the GAD7 Scale and DAS, respectively. This means that these two tests have a high positive rate, and both tests have good diagnostic power (27)(Table 4 and Figure 1). As observed in Table 4 and Figure 1, based on the cut-off points with the best balance, the GAD-7 Scale questionnaire has 61.9\% and 86.9\% sensitivity and specificity, respectively, with a Youden's index of 0.47 and 95\% confidence interval (CI) (79.4 - 92.5). Therefore, those who scored higher than 8 on the GAD-7 Scale questionnaire $(n=29)$ were recognized as patients. 
Table 3. Internal Consistency, Mean Scores Related to Questionnaires, and Convergent Validity

\begin{tabular}{|cc|}
\hline & Values \\
\hline Internal consistency $(\alpha)$ & 0.88 \\
\hline GAD-7 Scale & 0.74 \\
\hline Templer's DAS & \\
\hline Mean score \pm SD & $4.37 \pm 5.26$ \\
\hline GAD-7 Scale & $5.48 \pm 3.13$ \\
\hline DAS & \\
\hline CADvergent Validity (GAD-7 Scale and DAS) & \\
\hline $\begin{array}{l}\text { Anxiety Scale } \\
\text { Pearson correlation }\end{array}$ & 0.39 \\
\hline P-value & 0.000 \\
\hline
\end{tabular}

As displayed in Table 4, the sensitivity and specificity of the DAS questionnaire are $47.8 \%$ and $73.8 \%$, with $95 \%$ CI (65.2 - 81.2), respectively. Therefore, those who scored higher than 7 on DAS $(n=45)$ were regarded as patients (Table 4 and Figure 1).

\section{Discussion}

The reliability of both the GAD-7 Scale and DAS questionnaires was assessed using Cronbach's alpha and observed to be 0.88 and 0.74 , respectively. Based on the cut-off points with the best balance, the GAD-7 Scale and DAS can detect the patients with $61.9 \%$ and $47.8 \%$ accuracy, respectively. Furthermore, in this study, there was an acceptable Youden's index for the GAD-7 Scale (0.47) and DAS (0.2), but with poor +LR and -LR. Therefore, these tools cannot help diagnose or rule out the disease.

These two instruments have been translated into many languages across the globe (16, 28-31). For example, Spitzer et al. showed the cut-off point of $\geq 10$ as an optimal balance between sensitivity and specificity for the GAD (16). With this scoring for these tools, these results are not surprising given the comorbidity, severity, and acuity of the current sample. However, depending on the culture and target population, the cut-off points for the GAD-7 Scale (2) and DAS questionnaires were reported to be 8 and 7 (2), respectively, which are matched with the results of the present study $(16,32)$. The current study was conducted in a clinical setting. The patients accepted to have COVID19 , and only a clinical interview could help identify anxiety disorders with more accuracy.

These two scales were previously reported to have an acceptable level of internal consistency. The findings of the current study confirm the results of similar studies re- lated to the origin of these tools $(16,33,34)$ showing that they have excellent performance in the target population of Iran.

The female patients reported higher levels of generalized anxiety and death anxiety, compared to male patients. This finding is consistent with the results of other studies that have reported higher levels of COVID-19-related anxiety among female patients $(35,36)$. Female patients are at greater risk of psychological problems than male patients (37). Female patients have a stronger desire for knowledge about COVID-19. In addition, female patients have ovarian hormone fluctuations and endogenous estradiol changes across the menstrual cycle that intensify the effect of the aforementioned factors (38).

It was further observed that one in five participants reported the symptoms of generalized anxiety disorder, and one in three reported the symptoms of death anxiety. During pandemics, the number of individuals who are mentally affected exceeds those who contract that infectious disease. Pandemics deteriorate anxiety-related conditions. A study performed during the SARS outbreak reported that about one-third of participants showed anxiety disorders (39). The reports related to the previous pandemics also indicated that the mental health consequences of that event were higher and more persistent, compared to the number of affected patients $(40,41)$. In the outbreaks of communicable diseases, anxiety is a natural reaction to a lifethreatening condition. Therefore, physicians can distinguish between fear and anxiety disorder (42).

\subsection{Strengths and Limitations of Study}

The relative advantage of the present study lies in the fact that no clinical cut-off point has been established for inpatients in hospitals to date. However, the current study had some remarkable limitations. First, the major part of sampling in this study (about 90\%) was web-based, and there was no possibility to measure participation rates. Therefore, it can be argued that several patients were selectively included in the study. Accordingly, there is the possibility of selection bias in the present study. Another limitation is that the history of psychiatric illnesses, especially anxiety disorders, had not been assessed prior to the onset of the epidemic. Since the anxiety level is different in hospitalized and nonhospitalized COVID-19 patients, the obtained results are valid for hospitalized patients and not for all patients. The third limitation is concerned with the exclusion of divergent instrumentation due to patients' reluctance to participate in long surveys. Therefore, this point should be taken into account in future studies. Furthermore, anxiety varies over the course of diseases and hospitalization. Therefore, longitudinal studies are required to evaluate the degree of variability of anx- 


\begin{tabular}{|c|c|c|c|c|c|c|c|c|c|c|c|c|c|c|c|}
\hline Scale & $\begin{array}{l}\text { Area Under the } \\
\text { Curve }\end{array}$ & $\mathbf{S E}$ & CI & Sig & Criterion & Sensitivity & $95 \% \mathrm{CI}$ & Specificity & $95 \% \mathrm{CI}$ & $+\mathbf{L R}$ & - IR & $+\mathbf{P V}$ & $-\mathrm{PV}$ & DOR & Youden's Index \\
\hline GAD-7 Scale & 0.753 & 0.064 & $0.672-0.823$ & 0.0001 & $>8^{*}$ & 61.90 & $38.5-81.8$ & 86.96 & 79.4-92.5 & 4.75 & 0.44 & 46.4 & 92.6 & 0.47 & 0.47 \\
\hline DAS & 0.638 & 0.067 & $0.556-0.715$ & 0.0378 & $>7^{*}$ & 47.83 & $26.8-69.4$ & 73.81 & $65.2-81.2$ & 1.83 & 0.71 & 25.0 & 88.6 & 0.78 & 0.2 \\
\hline
\end{tabular}

Abbreviations: GAD-7, Generalized Anxiety Disorder 7-Item; DAS, Death Anxiety Scale; SE, standard error; CI, confidence interval; +LR, positive likelihood ratio; - $\mathrm{LR}$, negative likelihood ratio; +PV, positive predictive value; -PV, negative predictive value; DOR, diagnostic odds ratio

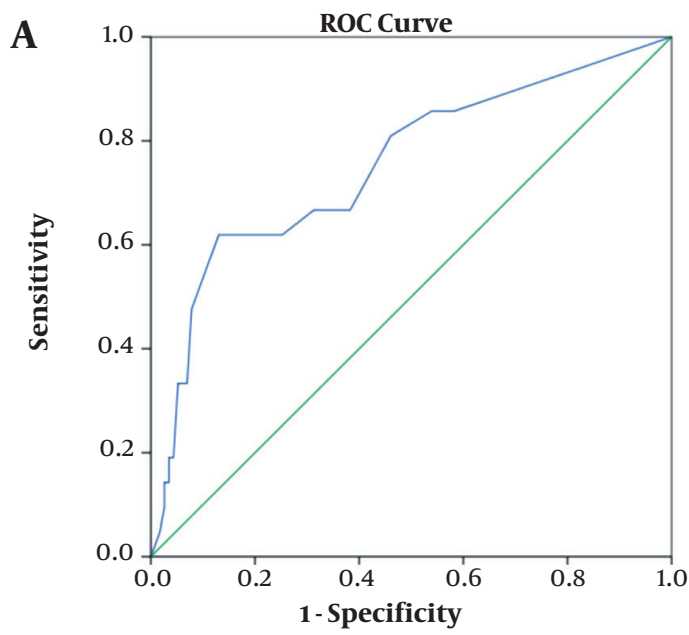

Diagonal Segments are Produced by Ties.

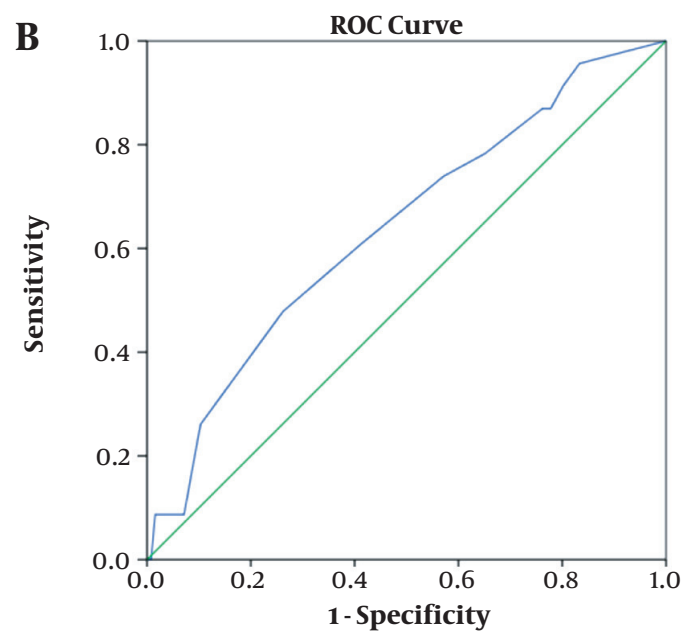

Diagonal Segments are Produced by Ties.

Figure 1. Area under the receiver operating characteristic curve for A) Generalized Anxiety Disorder 7-Item Scale and B) Death Anxiety Scale

iety disorders from the onset of disease to the time of recovery.

\subsection{Conclusions}

It will likely take a few years to bring the coronavirus under control worldwide (43). The GAD-7 Scale and DAS both showed adequate psychometric properties and diagnostic accuracy; therefore, they are applicable for anxiety screening in patients with COVID-19. The results of this study confirmed the validity of the GAD-7 Scale and DAS questionnaires for the assessment of dysfunctional coronavirus anxiety in the general Iranian population. With the Persian versions of the GAD-7 Scale and DAS, those psychologically affected by the pandemic can be effectively screened fast by mental professionals in Iran during hospitalization and after discharge to prevent the adverse mental health consequences of COVID-19.

\section{Acknowledgments}

The authors would like to express their gratitude to Velayat Hospital of Damghan, Iran, and Imam Khomeini Hospital of Mahabad, Iran, for their cooperation in collecting the data.

\section{Footnotes}

Authors' Contribution: Fattah and Babakhanian designed the study. Saeeidi, Darbani, Borumand, Setayesh, and Mohammadi collected the data. Fattah and Babakhanian wrote the final draft.

Conflict of Interests: The authors declare that there is no conflict of interest.

Ethical Approval: IR.MAZUMS.REC.1399.131; link: ethics.research.ac.ir/EthicsProposalView.php?id=130612

Funding/Support: This study was funded by the Student Research Committee of Mazandaran University of Medical Sciences.

\section{References}

1. Paraskevis D, Kostaki EG, Magiorkinis G, Panayiotakopoulos G, Sourvinos G, Tsiodras S. Full-genome evolutionary analysis of the novel corona virus (2019-nCoV) rejects the hypothesis of emergence as a result of a recent recombination event. Infect Genet Evol. 2020;79:104212. doi: 10.1016/j.meegid.2020.104212. [PubMed: 32004758]. [PubMed Central: PMC7106301].

2. Nicomedes CJC, Avila RMA. An analysis on the panic during COVID19 pandemic through an online form. J Affect Disord. 2020;276:14-22. doi: 10.1016/j.jad.2020.06.046. [PubMed:32697692]. [PubMed Central: PMC7362858]. 
3. Qiu J, Shen B, Zhao M, Wang Z, Xie B, Xu Y. A nationwide survey of psychological distress among Chinese people in the COVID-19 epidemic: implications and policy recommendations. Gen Psychiatr. 2020;33(2). e100213. doi: 10.1136/gpsych-2020-100213. [PubMed: 32215365]. [PubMed Central: PMC7061893].

4. Taylor S, Asmundson GJ. Treating health anxiety: A cognitive-behavioral approach. 11. Guilford Press; 2004.112 p.

5. Asmundson GJG, Taylor S. Coronaphobia: Fear and the 2019-nCoV outbreak. J Anxiety Disord. 2020;70:102196. doi: 10.1016/j.janxdis.2020.102196. [PubMed: 32078967]. [PubMed Central: PMC7134790].

6. Menzies RE, Neimeyer RA, Menzies RG. Death anxiety, loss, and grief in the time of COVID-19. Behav Change. 2020;37(3):111-5. doi: 10.1017/bec.2020.10.

7. Lee AM, Wong JG, McAlonan GM, Cheung V, Cheung C, Sham PC, et al. Stress and psychological distress among SARS survivors 1 year after the outbreak. Can J Psychiatry. 2007;52(4):233-40. doi: 10.1177/070674370705200405. [PubMed: 17500304].

8. Lee SM, Kang WS, Cho AR, Kim T, Park JK. Psychological impact of the 2015 MERS outbreak on hospital workers and quarantined hemodialysis patients. Compr Psychiatry. 2018;87:123-7. doi: 10.1016/j.comppsych.2018.10.003. [PubMed: 30343247]. [PubMed Central: PMC7094631].

9. Turkish Academy of Sciences. The assessment report on COVID-19 global outbreak. TÜRKEY: Turkish Academy of Sciences; 2020.

10. Taylor S. The psychology of pandemics: Preparing for the next global outbreak of infectious disease. Cambridge Scholars Publishing; 2019.

11. Ransing R, Ramalho R, Orsolini L, Adiukwu F, Gonzalez-Diaz JM, Larnaout A, et al. Can COVID-19 related mental health issues be measured Assessment options for mental health professionals. Brain Behav Immun. 2020;88:32-4. doi: 10.1016/j.bbi.2020.05.049. [PubMed: 32470593]. [PubMed Central: PMC7248629].

12. Kaviani H, Seyfourian H, Sharifi V, Ebrahimkhani N. [Reliability and validity of Anxiety and Depression Hospital Scales (HADS): Iranian patients with anxiety and depression disorders]. Tehran Univ Med J. 2009;67(5):379-85. Persian.

13. Power M, Fell G, Wright M. Principles for high-quality, high-value testing. Evid Based Med. 2013;18(1):5-10. doi: 10.1136/eb-2012-100645. [PubMed: 22740357]. [PubMed Central: PMC3585491].

14. Mina FB, Billah M, Karmakar S, Das S, Rahman MS, Hasan MF, et al. An online observational study assessing clinical characteristics and impacts of the COVID-19 pandemic on mental health: a perspective study from Bangladesh. Z Gesundh Wiss. 2021:1-9. doi: 10.1007/s10389020-01445-2. [PubMed: 33432286]. [PubMed Central: PMC7787928].

15. Bujang MA, Adnan TH. Requirements for minimum sample size for sensitivity and specificity analysis. J Clin Diagn Res. 2016;10(10):YE016. doi: 10.7860/JCDR/2016/18129.8744. [PubMed: 27891446]. [PubMed Central: PMC5121784].

16. Spitzer R, Kroenke K, Williams JB, Löwe B. A brief measure for assessing generalized anxiety disorder: the GAD-7. Arch Intern Med. 2006;166(10):1092-7. doi: 10.1001/archinte.166.10.1092. [PubMed: 16717171].

17. Naeinian M, SHAIRI M, Sharifi M, Hadian M. [To study reliability and validity for a brief measure for assessing Generalized Anxiety Disorder (GAD-7)]. Clin Psychol Pers. 2011;2(4):41-50. Persian.

18. Templer DI, Ruff CF. Death Anxiety Scale means, standard deviations, and embedding. Psychol Rep. 2016;29(1):173-4. doi: 10.2466/pro.1971.29.1.173.

19. First MB, Williams JB, Karg RS, Spitzer RL. User's guide for the SCID-5-CV structured clinical interview for DSM-5® disorders: Clinical version. American Psychiatric Publishing; 2016.

20. Wong HB, Lim GH. Measures of diagnostic accuracy: Sensitivity, specificity, PPV and NPV. Proc Singapore Healthc. 2011;20(4):316-8.

21. Fletcher GS. Clinical epidemiology: The essentials. 5 th ed. Lippincott Williams \& Wilkins publisher; 2013.

22. Shreffler J, Huecker MR. Diagnostic testing accuracy: Sensitivity, speci- ficity, predictive values and likelihood ratios. Treasure Island (FL): StatPearls Publishing; 2020.

23. Khatami A. [Evaluation of diagnostic test accuracy studies]. J Cosmet Dermato. 2011;2(1):47-59. Persian.

24. Wikipedia. Youden's J statistic. 2021. Available from: https://en. wikipedia.org/wiki/Youden\%27s_J_statistic.

25. Fluss R, Faraggi D, Reiser B. Estimation of the Youden Index and its associated cutoff point. Biom J. 2005;47(4):458-72. doi: 10.1002/bimj.200410135. [PubMed: 16161804].

26. Martin JL, Williams KS, Abrams KR, Turner DA, Sutton AJ, Chapple $\mathrm{C}$, et al. Systematic review and evaluation of methods of assessing urinary incontinence. Health Technol Assess. 2006;10(6):1-132. doi: 10.3310/hta10060. [PubMed: 16487456].

27. Wixted JT, Mickes L, Wetmore SA, Gronlund SD, Neuschatz JS. ROC analysis in theory and practice. J Appl Res Mem Cogn. 2017;6(3):343-51. doi:10.1016/j.jarmac.2016.12.002.

28. Budikayanti A, Larasari A, Malik K, Syeban Z, Indrawati LA, Octaviana F. Screening of generalized anxiety disorder in patients with epilepsy: Using a valid and reliable Indonesian version of generalized anxiety disorder-7 (GAD-7). Neurol Res Int. 2019;2019:5902610. doi: 10.1155/2019/5902610. [PubMed: 31275648]. [PubMed Central: PMC6582805].

29. Sousa TV, Viveiros V, Chai MV, Vicente FL, Jesus G, Carnot MJ, et al. Reliability and validity of the Portuguese version of the Generalized Anxiety Disorder (GAD-7) scale. Health Qual Life Outcomes. 2015;13:50. doi: 10.1186/s12955-015-0244-2. [PubMed: 25908249]. [PubMed Central: PMC4424548].

30. Garcia-Campayo J, Zamorano E, Ruiz MA, Pardo A, Perez-Paramo M, Lopez-Gomez V, et al. Cultural adaptation into Spanish of the generalized anxiety disorder-7 (GAD-7) scale as a screening tool. Health Qual Life Outcomes. 2010;8:8. doi:10.1186/1477-7525-8-8. [PubMed: 20089179]. [PubMed Central: PMC2831043].

31. Plummer F, Manea L, Trepel D, McMillan D. Screening for anxiety disorders with the GAD-7 and GAD-2: a systematic review and diagnostic metaanalysis. Gen Hosp Psychiatry. 2016;39:24-31. doi: 10.1016/j.genhosppsych.2015.11.005. [PubMed: 26719105].

32. Gonen G, Kaymak SU, Cankurtaran ES, Karslioglu EH, Ozalp E, Soygur $\mathrm{H}$. The factors contributing to death anxiety in cancer patients. J Psychosoc Oncol. 2012;30(3):347-58. doi: 10.1080/07347332.2012.664260. [PubMed: 22571248].

33. Lopez-Castedo A, Gonzalez-Rodriguez R, Vazquez Perez R. [Psychometric properties of the Death Anxiety Scale in patients with ischemic cardiomyopathy]. Rev Esp Salud Publica. 2019;93. [PubMed: 31576814].

34. Mirhosseini S, Dadgari A, Basirinezhad M, Mohammadpourhodki R, Ebrahimi $\mathrm{H}$. The proportion of death anxiety and its related factors during the COVID-19 pandemic in the Iranian population. Fam Med Prim Care Rev. 2021;23(1):36-40. doi: 10.5114/fmpcr.2021.103154.

35. Ahmed O, Faisal RA, Sharker T, Lee SA, Jobe MC. Adaptation of the Bangla version of the COVID-19 Anxiety Scale. Int J Ment Health Addict. 2020:1-12. doi: 10.1007/s11469-020-00357-2. [PubMed: 32837436]. [PubMed Central: PMC7320841].

36. Wang C, Pan R, Wan X, Tan Y, Xu L, Ho CS, et al. Immediate psychological responses and associated factors during the initial stage of the 2019 coronavirus disease (COVID-19) epidemic among the general population in China. Int J Environ Res Public Health. 2020;17(5). doi: 10.3390/ijerph17051729. [PubMed: 32155789]. [PubMed Central: PMC7084952].

37. Hou F, Bi F, Jiao R, Luo D, Song K. Gender differences of depression and anxiety among social media users during the COVID-19 outbreak in China:a cross-sectional study. BMC Public Health. 2020;20(1):1648. doi: 10.1186/s12889-020-09738-7. [PubMed: 33148202]. [PubMed Central: PMC7609822].

38. Yan S, Xu R, Stratton TD, Kavcic V, Luo D, Hou F, et al. Sex differences and psychological stress: Responses to the COVID-19 pandemic in China. BMC Public Health. 2021;21(1):79. doi: 10.1186/s12889-020-10085- 
w. [PubMed: 33413224]. [PubMed Central: PMC7789895].

39. Su TP, Lien TC, Yang CY, Su YL, Wang JH, Tsai SL, et al. Prevalence of psychiatric morbidity and psychological adaptation of the nurses in a structured SARS caring unit during outbreak: a prospective and periodic assessment study in Taiwan.J Psychiatr Res. 2007;41(1-2):119-30. doi: 10.1016/j.jpsychires.2005.12.006. [PubMed: 16460760]. [PubMed Central: PMC7094424].

40. Shigemura J, Ursano RJ, Morganstein JC, Kurosawa M, Benedek DM. Public responses to the novel 2019 coronavirus (2019-nCoV) in Japan: Mental health consequences and target populations. Psychiatry Clin Neurosci.2020;74(4):281-2. doi:10.1111/pcn.12988.[PubMed:32034840].
[PubMed Central: PMC7168047].

41. Reardon S. Ebola's mental-health wounds linger in Africa. Nature. 2015;519(7541):13-4. doi: 10.1038/519013a. [PubMed: 25739606].

42. Tzur Bitan D, Grossman-Giron A, Bloch Y, Mayer Y, Shiffman N, Mendlovic S. Fear of COVID-19 Scale: Psychometric characteristics, reliability and validity in the Israeli population. Psychiatry Res. 2020;289:113100. doi: 10.1016/j.psychres.2020.113100.

43. The Lancet Microbe. COVID-19 vaccines: The pandemic will not end overnight. Lancet Microbe. 2021;2(1). e1. doi: 10.1016/S26665247(20)30226-3. [PubMed: 33521732]. [PubMed Central: PMC7831782]. 